\title{
A SIMPLE PROOF OF THE UNIQUENESS OF PERIODIC ORBITS IN THE $1: 3$ RESONANCE PROBLEM
}

\author{
SHIU-NEE CHOW, CHENGZHI LI, AND DUO WANG
}

(Communicated by Kenneth R. Meyer)

\begin{abstract}
In 1979, E. Horozov considered the versal deformation of a planar vector field which is invariant under a rotation through an angle $2 \pi / 3$ (with resonance of order 3 ). In his study, the most difficult part of the proof is on the uniqueness of limit cycles. In this note we give a simple and elementary (without the theory of algebraic geometry proof of the uniqueness of periodic orbits in the $1: 3$ resonance problem.
\end{abstract}

In [6], Horozov considered the versal deformation of a planar vector field which is invariant under a rotation through an angle $2 \pi / 3$ (with resonance of order 3). The main difficulty in [6] is to prove the uniqueness of limit cycles. In this note we give a simpler proof of the uniqueness result by using the PicardFuchs equation and a specific technique introduced by Carr, Chow, and Hale [2] (see Proposition 4). Our proof is elementary and does not need results from algebraic geometry (see [6]).

Consider a family of vector fields with resonance of order 3. It is well known that a normal form equation of order 3 is given by the following equation (see, for example, Arnold [1] p. 293 or [7]):

$$
\dot{z}=\varepsilon z+A z|z|^{2}+\bar{z}^{2},
$$

where $z, A \in \mathbb{C}$, and $\varepsilon=\varepsilon_{1}+i \varepsilon_{2}$ is a complex parameter. For $\varepsilon=0$, (1) becomes

$$
\dot{z}=A z|z|^{2}+\bar{z}^{2} .
$$

The following could be found in [6].

Theorem A. If $\operatorname{Re} A=a \neq 0$, then

(a) (1) is a versal deformation of (2) with resonance of order 3;

Received by the editors February 5, 1988 and, in revised form, May 8, 1988.

1980 Mathematics Subject Classification (1985 Revision). Primary 58F14.

Key words and phrases. Bifurcation diagram, phase portrait, periodic orbit, Picard-Fuchs equation.

Shui-Nee Chow partially supported by DARPA.

(C) 1989 American Mathematical Society $0002-9939 / 89 \$ 1.00+\$ .25$ per page 
(b) the bifurcation diagram of (1) consists of the origin and following curves in parameter space (Figure 1):

$$
\begin{gathered}
H^{ \pm}=\left\{\varepsilon \mid \varepsilon_{1}=0, \varepsilon_{2} \neq 0\right\}, \\
H L^{ \pm}=\left\{\varepsilon \mid \varepsilon_{1}=-\frac{a}{8} \varepsilon_{2}^{2}+0\left(\left|\varepsilon_{2}\right|^{3}\right), \varepsilon_{2} \neq 0\right\} ;
\end{gathered}
$$

(c) the phase portraits of (1) for $\varepsilon$ in various regions in parameter space are shown in Figure 1.

Remarks. (1) In Theorem A, $H$ and $H L$ are curves along which Hopf bifurcation and heteroclinic loop bifurcation occur. (2) In [6] there is a misprint in the equation for $H L$ (see [6, p. 187]).

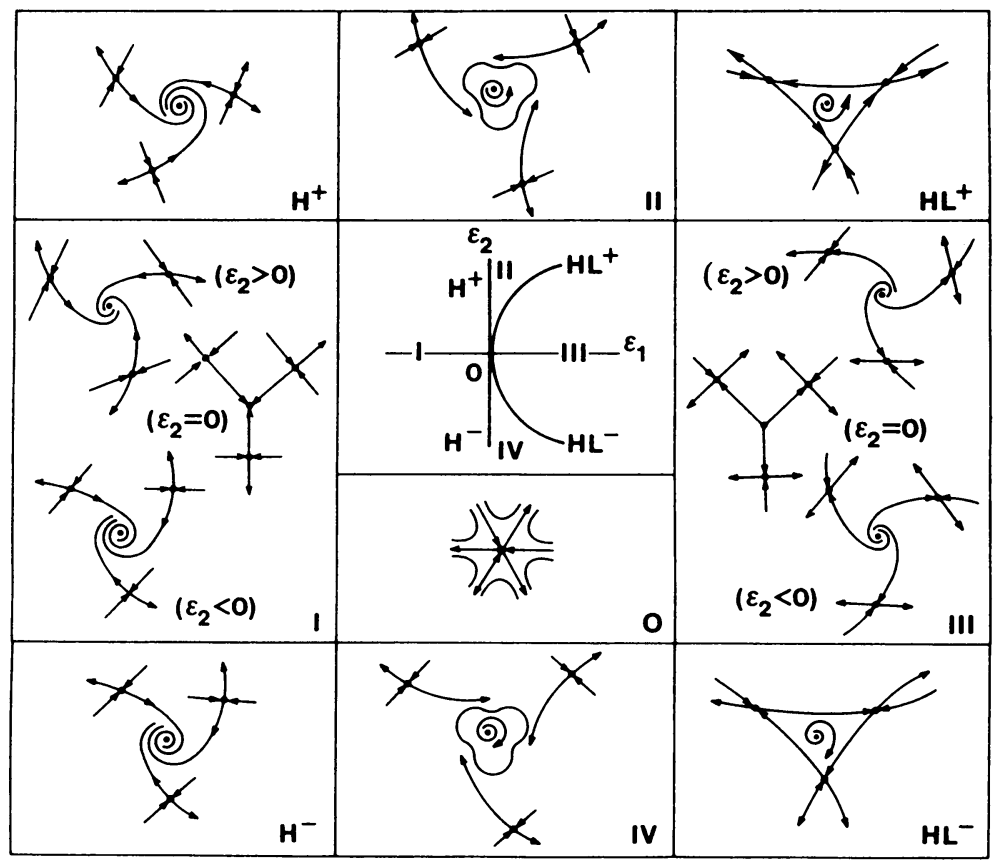

FIGURE 1.

We note that limit cycles appear only in regions II and IV in Figure 1. The difficulty in proving Theorem A is to verify the uniqueness of limit cycles in these regions. In the following, we give a proof of the uniqueness result. For a complete proof of Theorem A, we refer the reader to Horozov [6] or Chow, $\mathrm{Li}$, and Wang [3].

In order to prove that equation (1) is a versal deformation of equation (2), Horozov [6] considered a planar system with $Z_{3}$-symmetry in a small neighborhood of the origin $z=0$ :

$$
\dot{z}=\mu z+A(\mu) z|z|^{2}+\bar{z}^{2}+O\left(|z|^{4}\right),
$$


where $\mu=\mu_{1}+i \mu_{2}$ is a complex parameter. It is shown in [6] that the bifurcation diagram and phase portraits are independent of the higher order terms $O\left(|z|^{4}\right)$ in equation (3). It is not difficult to show that equation (3) has four equilibria in a small neighborhood of phase space. Furthermore, one of the equilibria, $z=0$, is always a focus or node, and the others are always saddles (see Figure 1).

By a symplectic transformation

(3) is transformed to the following:

$$
\left\{\begin{array}{l}
x=\sqrt{2 \rho} \cos \varphi \\
y=\sqrt{2 \rho} \sin \varphi
\end{array}\right.
$$

$$
\left\{\begin{array}{l}
\dot{\rho}=\mu_{1} 2 \rho+a(\mu)(2 \rho)^{2}+(2 \rho)^{3 / 2} \cos 3 \varphi+(2 \rho)^{5 / 2} F_{1}(\rho, 3 \varphi, \mu), \\
\dot{\varphi}=\mu_{2}+b(\mu) 2 \rho-(2 \rho)^{1 / 2} \sin 3 \varphi+(2 \rho)^{3 / 2} F_{2}(\rho, 3 \varphi, \mu),
\end{array}\right.
$$

where $\left.F_{j}\right|_{\mu=0}=0, \quad F_{j}$ is $2 \pi$-periodic with respect to $\varphi, j=1,2$, $a(\mu)=\operatorname{Re} A(\mu), b(\mu)=\operatorname{Im} A(\mu),|\rho|<\delta_{1},|\mu|<\delta_{1}$ and $\delta_{1}>0$ is sufficiently small. We suppose $a(\mu)<0$ below. The case of $a(\mu)>0$ is similar.

Let

$$
\mu_{1}=-\frac{1}{a(\mu)} \delta^{2} \beta, \mu_{2}=-\frac{1}{a(\mu)} \delta, \rho \rightarrow \frac{\delta^{2}}{a^{2}(\mu)} \rho, b(\mu) \rightarrow-a(\mu) b(\mu), t \rightarrow-\frac{a(\mu) t}{\delta},
$$

where $\delta$ and $\beta$ are parameters, $\delta$ is small and $\beta \in(-\infty, \infty)$. Hence, (4) becomes

$$
\left\{\begin{array}{l}
\dot{\rho}=\delta \beta(2 \rho)-\delta(2 \rho)^{2}+(2 \rho)^{3 / 2} \cos 3 \varphi+\delta^{2}(2 \rho)^{5 / 2} \bar{F}_{1}, \\
\dot{\varphi}=1+b \delta(2 \rho)-(2 \rho)^{1 / 2} \sin 3 \varphi+\delta^{2}(2 \rho)^{3 / 2} \bar{F}_{2} .
\end{array}\right.
$$

Suppose that $\left(\rho_{o}(\delta, \beta), \varphi_{o}(\delta, \beta)\right)$ is an equilibrium of $(6)$ which is different from the origin. Let

$$
\left\{\begin{array}{l}
r=\frac{\rho}{2 \rho_{\prime \prime}}, \\
\theta=\frac{\pi}{6}+\varphi-\varphi_{o}=\varphi-\psi(\beta, \delta) .
\end{array}\right.
$$

Then (6) takes the form

$$
\left\{\begin{array}{l}
\dot{r}=\delta \beta(2 r)-\delta\left(2 \rho_{o}\right)(2 r)^{2}+\left(2 \rho_{o}\right)^{1 / 2}(2 r)^{3 / 2} \cos 3(\theta+\psi)+\delta^{2}(2 r)^{5 / 2} \widetilde{F}_{1} \\
\dot{\theta}=1+\delta\left(2 \rho_{o}\right) b(2 r)-\left(2 \rho_{o}\right)^{1 / 2}(2 r)^{1 / 2} \sin 3(\theta+\psi)+\delta^{2}(2 r)^{3 / 2} \widetilde{F}_{2} .
\end{array}\right.
$$

Let $\widetilde{H}(\delta, r, \theta)$ denote the right-hand side of the equation of $\dot{\theta}$ in (7). We note that the coordinates of the equilibria of (7) are independent of $\delta$ and $\beta$ and these equilibria are: $r=0$ and $\left(r_{k}, \theta_{k}\right)$, where $r_{k}=\frac{1}{2}, \theta_{k}=\pi / 6+2 k \pi / 3$, $k=0,1,2$.

For $\delta=0$, we have $2 \rho_{o}=1$ and (7) is a Hamiltonian system

$$
\left\{\begin{array}{l}
\dot{r}=(2 r)^{3 / 2} \cos 3 \theta \\
\dot{\theta}=1-(2 r)^{1 / 2} \sin 3 \theta
\end{array}\right.
$$


with the first integral

$$
H(r, \theta)=r-\frac{1}{3}(2 r)^{3 / 2} \sin 3 \theta
$$

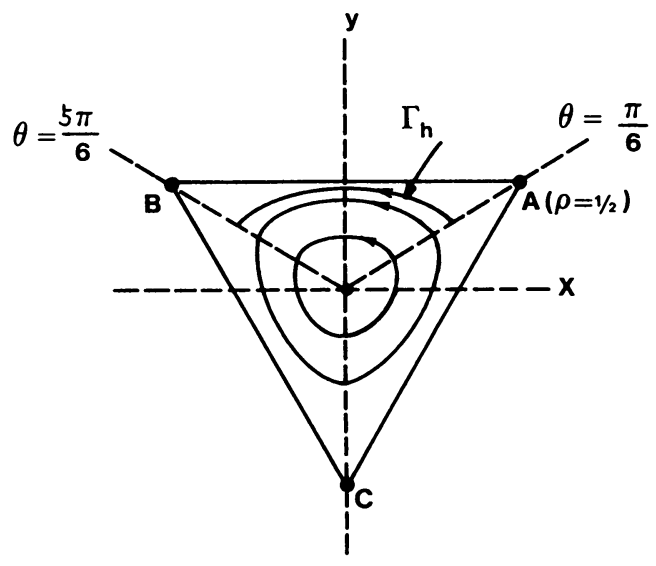

Figure 2.

The level curves of $H=h$ are shown in Figure 2, where $0 \leq h \leq \frac{1}{6} . H=0$ corresponds to the equilibrium $r=0$, and $H=\frac{1}{6}$ corresponds to the three heteroclinic orbits.

Obviously, any closed orbit of (7) must surround the origin and cross the line segment

$$
L=\left\{(r, \theta) \mid \theta=\pi / 6,0 \leq r \leq \frac{1}{2}\right\}
$$

Let

$$
H^{\delta}(r, \theta)=\int_{0}^{r} \tilde{H}(\delta, r, \theta) d r .
$$

Then (7) can be rewritten in the form:

$$
\left\{\begin{array}{l}
\dot{r}=-\frac{\partial H^{\delta}}{\partial \theta}+2 \delta r\left[\beta-\left(2 \rho_{0}\right)(2 r)+\delta(2 r)^{3 / 2} \bar{F}\right], \\
\dot{\theta}=\frac{\partial H^{\delta}}{\partial r} .
\end{array}\right.
$$

We note that $H^{\delta}(r, \pi / 6)$ is monotone in $r$ if $0 \leq r \leq \frac{1}{2}$. Thus, we parameterize $L$ by $H(r, \pi / 6)=h$. Let $\Gamma$ be a trajectory of (7) starting from a point on $L$ and later intersecting the half line $\theta=5 \pi / 6$. We denote by $\Gamma(\delta, h, \beta)$ the part of $\Gamma$ which lies between the half lines $\theta=\pi / 6$ and $\theta=5 \pi / 6$. Since (7) is invariant under a rotation through an angle $2 \pi / 3, \Gamma$ is a closed orbit of (7) if and only if

$$
\int_{\Gamma(\delta, h, \beta)}\left(\frac{d H^{\delta}}{d t}\right) d t=0
$$


It is easy to obtain from (10) that for $\delta \neq 0$ (11) is equivalent to

$$
\Phi(\delta, h, \beta) \equiv \int_{\Gamma(\delta, h, \beta)} r\left[\beta-\left(2 \rho_{0}\right)(2 r)+\delta(2 r)^{3 / 2} \bar{F}\right] d \theta=0 .
$$

Let $\Gamma_{h}$ be the part of level curve $H=h, 0 \leq h \leq 1 / 6, \pi / 6 \leq \theta \leq 5 \pi / 6$. Define

$$
I_{k}(h)=\int_{\Gamma_{h}} r^{k} d \theta, \quad k=1,2,3 .
$$

In terms of $I_{k}(h) \quad(k=1,2)$, we have

$$
\Phi(0, h, \beta)=\int_{\Gamma_{h}} r(\beta-2 r) d \theta=\beta I_{1}(h)-2 I_{2}(h) .
$$

Obviously, $I_{1}(h)>0$ for $0<h<\frac{1}{6}$ and

$$
\lim _{h \rightarrow 0} \frac{I_{2}(h)}{I_{1}(h)}=0 \text {. }
$$

Let

$$
p(h)=\frac{I_{2}(h)}{I_{1}(h)}, \quad 0 \leq h \leq \frac{1}{6} .
$$

By using similar arguments as in $[2,4,5,8$, or 9] one obtains that the uniqueness of limit cycles of (7) is equivalent to the monotonicity of $p(h), 0 \leq h \leq \frac{1}{6}$. We will prove the monotonicity of $p(h)$ in Proposition 4 below. The following lemmas are needed.

Lemma 1. $p(h)$ satisfies the following equation

(14) $9 h(6 h-1) p^{\prime}(h)=-12 p^{2}+(28 h-\varphi(h)+9) p+48 h^{2}-18 h+6 h \varphi(h)$, where $0<h<\frac{1}{6}$ and

$$
\varphi(h)=6 h^{2}(6 h-1) \frac{I_{1}^{\prime \prime}(h)}{I_{1}(h)} .
$$

Proof. Suppose that the function $r=r(\theta, h)$ is defined by $H(r, \theta)=h$ for $\pi / 6 \leq \theta \leq 5 \pi / 6$ and $0 \leq h \leq \frac{1}{6}$. From (9) and $H(r, \theta)=h$, we have that

$$
\frac{\partial r}{\partial h}=\frac{1}{1-\sqrt{2 r} \sin 3 \theta}=\frac{2 r}{3 h-r}>0, \quad 0<h<\frac{1}{6} .
$$

The above expression is positive because $0<r<\frac{1}{2}$. Hence,

$$
I_{k}^{\prime}(h)=2 k \int_{\Gamma_{h}} \frac{r^{k}}{3 h-r} d \theta
$$

Obviously,

$$
I_{k}(h)=\int_{\Gamma_{h}} \frac{r^{k}(3 h-r)}{3 h-r} d \theta=\frac{3 h}{2 k} I_{k}^{\prime}-\frac{1}{2(k+1)} I_{k+1}^{\prime} .
$$

In particular

$$
\left\{\begin{array}{l}
I_{1}=\frac{3}{2} h I_{1}^{\prime}-\frac{1}{4} I_{2}^{\prime}, \\
I_{2}=\frac{3}{4} h I_{2}^{\prime}-\frac{1}{6} I_{3}^{\prime} .
\end{array}\right.
$$


From (9), we have

$$
I_{3}(h)=\int_{\Gamma_{h}} r^{3} d \theta=\frac{9}{8} \int_{\Gamma_{h}} \frac{(r-h)^{2}}{\sin ^{2} 3 \theta} d \theta=-\frac{3}{8} \int_{\pi / 6}^{5 \pi / 6}(r-h)^{2} d(\cot \theta) .
$$

Integrating by parts and using (15), (9), and (8), we have

$$
\begin{aligned}
I_{3} & =\frac{3}{4} \int_{\Gamma_{h}} \frac{r-h}{\sin 3 \theta} \frac{(2 r)^{3 / 2} \cos ^{2} 3 \theta}{1-\sqrt{2 r} \sin 3 \theta} d \theta \\
& =\frac{1}{2} \int_{\Gamma_{h}} \frac{r\left[(2 r)^{3}-9(r-h)^{2}\right]}{3 h-r} d \theta \\
& =-4 I_{3}+\left(\frac{9}{2}-12 h\right) I_{2}+\left(\frac{9}{2} h-36 h^{2}\right) I_{1}+\left(54 h^{3}-9 h^{2}\right) I_{1}^{\prime} .
\end{aligned}
$$

Hence

$$
I_{3}=\left(\frac{9}{10}-\frac{12}{5} h\right) I_{2}+\left(\frac{9}{10} h-\frac{36}{5} h^{2}\right) I_{1}+\left(\frac{54}{5} h^{3}-\frac{9}{5} h^{2}\right) I_{1}^{\prime} .
$$

Substituting (17) into (16), we have

$$
\left\{\begin{array}{l}
4 I_{1}=6 h I_{1}^{\prime}-I_{2}^{\prime}, \\
48 I_{2}=\left(18 h-48 h^{2}\right) I_{1}^{\prime}+(-9+44 h) I_{2}^{\prime}-24 h^{2}(6 h-1) I_{1}^{\prime \prime} .
\end{array}\right.
$$

Equation (18) is equivalent to

$$
\left\{\begin{array}{l}
9 h(6 h-1) I_{1}^{\prime}=(-9+44 h) I_{1}+12 I_{2}+6 h^{2}(6 h-1) I_{1}^{\prime \prime}, \\
9 h(6 h-1) I_{2}^{\prime}=\left(-18 h+48 h^{2}\right) I_{1}+72 h I_{2}+36 h^{3}(6 h-1) I_{1}^{\prime \prime},
\end{array}\right.
$$

for $0<h<\frac{1}{6}$. By (19) and the following

$$
p^{\prime}(h)=\frac{I_{2}^{\prime} I_{1}-I_{1}^{\prime} I_{2}}{I_{1}^{2}}
$$

we obtain (14). This proves the lemma.

Lemma 2. $\lim _{h \rightarrow 0} p^{\prime}(h)=1$.

Proof. As $h \rightarrow 0, I_{1}=O(h), I_{2}=O\left(h^{2}\right)$ (see (15)). Hence, $p(h)=O(h)$ and

$$
\begin{gathered}
I_{1}^{\prime \prime}(h)=6 \int_{\Gamma_{h}} \frac{r(r-h)}{(3 h-r)^{3}} d \theta=O\left(|h|^{-1 / 2}\right) \\
I_{1}^{\prime \prime \prime}(h)=12 \int_{\Gamma_{h}} \frac{r(r-h)(5 r-6 h)}{(3 h-r)^{5}} d \theta=O\left(|h|^{-3 / 2}\right) .
\end{gathered}
$$

Thus, $\varphi(h)=O\left(|h|^{1 / 2}\right), \varphi^{\prime}(h)=O\left(|h|^{-1 / 2}\right)$. Using the above estimates and L'Hospital's rule, we obtain from (14) $\lim _{h \rightarrow 0} p^{\prime}(h)=1$.

Lemma 3. $p\left(\frac{1}{6}\right)=\frac{1}{16}, p^{\prime}\left(\frac{1}{6}\right)=2 \sqrt{5} \ln ((3+\sqrt{5}) / 2)-4$.

Proof. $\Gamma_{1 / 6}$ is a curve given by $\{(r, \theta) \mid \sqrt{2 r} \sin \theta=1 / 4, \pi / 6 \leq \theta \leq 5 \pi / 6\}$. A direct calculation shows

$$
I_{1}\left(\frac{1}{6}\right)=\int_{\Gamma_{1 / 6}} r d \theta=\frac{\sqrt{3}}{16}, \quad I_{2}\left(\frac{1}{6}\right)=\int_{\Gamma_{1 / 6}} r^{2} d \theta=\frac{\sqrt{3}}{256},
$$


PERIODIC ORBITS IN THE $1: 3$ RESONANCE PROBLEM

1031

$$
I_{1}^{\prime}\left(\frac{1}{6}\right)=\int_{\Gamma_{1 / 6}} \frac{2 r}{\frac{1}{2}-r} d \theta=\frac{2}{\sqrt{15}} \ln \frac{3+\sqrt{5}}{2},
$$

and

$$
I_{2}^{\prime}\left(\frac{1}{6}\right)=\int_{\Gamma_{1 / 6}} \frac{4 r^{2}}{\frac{1}{2}-r} d \theta=\frac{\sqrt{3}}{4}\left[\frac{8 \sqrt{5}}{15} \ln \frac{3+\sqrt{5}}{2}-1\right] \text {. }
$$

Hence

$$
\begin{gathered}
p(1 / 6)=\left.\frac{I_{2}}{I-1}\right|_{h=1 / 6}=\frac{1}{16} \\
p^{\prime}(1 / 6)=\left.\frac{I_{2}^{\prime} I_{1}-I_{1}^{\prime} I_{2}}{I_{1}^{2}}\right|_{h=1 / 6}=2 \sqrt{5} \ln \frac{3+\sqrt{5}}{2}-4 .
\end{gathered}
$$

Proposition 4. $p^{\prime}(h)>0$ for $0<h<\frac{1}{6}$.

Proof. We will prove that if there exists $h_{0} \in(0,1 / 6)$ such that $p^{\prime}\left(h_{0}\right)=0$, then $p^{\prime \prime}\left(h_{0}\right)>0$. This is a contradiction since $p(0)=0$, $p^{\prime}(0)=1$ (Lemma 1).

Let

$$
Q(h)=I_{2}^{\prime}(h) / I_{1}^{\prime}(h), \quad 0<h<\frac{1}{6} .
$$

Then $p\left(h_{0}\right)=Q\left(h_{0}\right)$ and

$$
p^{\prime \prime}\left(h_{0}\right)=\left(I_{1}^{\prime}\left(h_{0}\right) / I_{1}\left(h_{0}\right)\right) Q^{\prime}\left(h_{0}\right)
$$

provided $p^{\prime}\left(h_{0}\right)=0$ (see Carr, Chow, and Hale [2]).

From the first equation of (18), we have

$$
6 h I_{1}^{\prime \prime}=I_{2}^{\prime \prime}-2 I_{1}^{\prime} \text {. }
$$

Hence

$$
\frac{I_{1}^{\prime \prime}}{I_{1}^{\prime}}=\frac{1}{6 h}\left(\frac{I_{2}^{\prime \prime}}{I_{1}^{\prime}}-2\right)=\frac{1}{6 h}\left(\frac{I_{2}^{\prime \prime}}{I_{2}^{\prime}} Q-2\right) \text {. }
$$

This implies

$$
Q^{\prime}(h) \equiv Q\left(\frac{I_{2}^{\prime \prime}}{I_{2}^{\prime}}-\frac{I_{1}^{\prime \prime}}{I_{1}^{\prime}}\right)=\frac{Q}{6 h}\left[(6 h-Q) \frac{I_{2}^{\prime \prime}}{I_{2}^{\prime}}+2\right] .
$$

The first equation of (18) implies $6 h-Q=4 I_{1} / I_{1}^{\prime}$.

If $p^{\prime}\left(h_{0}\right)=0\left(0<h_{0}<\frac{1}{6}\right)$, then $Q\left(h_{0}\right)=p\left(h_{0}\right)>0$ and

$$
\frac{I_{1}\left(h_{0}\right)}{I_{1}^{\prime}\left(h_{0}\right)}=\frac{I_{2}\left(h_{0}\right)}{I_{2}^{\prime}\left(h_{0}\right)} \text {. }
$$

Thus

$$
Q^{\prime}\left(h_{0}\right)=\left.\frac{Q(h)}{3 h}\left(\frac{2 I_{2} I_{2}^{\prime \prime}}{I_{2}^{\prime 2}}+1\right)\right|_{h=h_{0}}>0
$$

because

$$
I_{2}\left(h_{0}\right)=\int_{\Gamma_{h_{0}}} r^{2} d \theta>0,
$$




$$
\begin{gathered}
I_{2}^{\prime}\left(h_{0}\right)=\int_{\Gamma_{h_{0}}} \frac{4 r^{2}}{3 h_{0}-r} d \theta>0, \\
I_{2}^{\prime \prime}\left(h_{0}\right)=\int_{\Gamma_{h_{0}}} \frac{4 r^{2}\left(3 h_{0}+r\right)}{\left(3 h_{0}-r\right)^{3}} d \theta>0 .
\end{gathered}
$$

The fact that along $\Gamma_{h_{0}}, 3 h_{0}-r>0$ can be found from (15). This proves Proposition 4.

Remark. We note that all the above discussions are independent of the higher order terms $O\left(|z|^{4}\right)$.

\section{REFERENCES}

1. V. I. Arnold, Geometrical methods in the theory of ordinary differential equations, SpringerVerlag, New York, 1983.

2. J. Carr, S.-N. Chow, and J. K. Hale, Abelian integrals and bifurcation theory, J. Differential Equations 59 (1985), 413-436.

3. S.-N. Chow, C. Li, and D. Wang, Normal forms and bifurcation of vector fields (to appear).

4. R. H. Cushman and J. A. Sanders, A codimension two bifurcation with a third order PicardFuchs equation, J. Differential Equations 59 (1985), 243-256.

5. B. Drachman, S. van Gils, and Zhang Zhifen, Abelian integrals for quadratic vector fields, J. Reine Angew. Math. 382 (1987), 165-180.

6. E. I Horozov, Versal deformation of equivariant vector fields in the case of symmetry of order 2 and 3, Trans. of Petrovski Seminar, 5 (1979), 163-192, (In Russian).

7. C. Rousseau, Codimension 1 and 2 bifurcations of fixed points of diffeomorphisms and of periodic solutions of vector fields, 1987 (preprint).

8. R. H. Cushman and J. A. Sanders, Abelian integrals and global Hopf bifurcations, Dynamical Systems and Bifurcations (B. L. J. Braaksma, H. W. Broer, and F. Takens, editors) Lecture Notes in Mathematics 1125, Springer-Verlag, 1985, pp. 87-98.

9. Yu. S., II' yashenko, Zeros of special Abelian integrals in a real domain, Functional Anal. Appl. 11 (1977), 309-311.

Center for Dynamical Systems and Nonlinear Studies, Georgia Institute of TechNOLOGy, AtLanta, Georgia 30332

Current address: (Chengzhi Li): Department of Mathematics, Peking University, Bejing, China

Current address: (Duo Wang): Department of Mathematics, Peking University, BEIJING, ChINA 